Check for updates

Cite this: RSC Adv., 2017, 7, 54416

Received 7th September 2017

Accepted 22nd November 2017

DOI: $10.1039 / \mathrm{c} 7 \mathrm{ra09979g}$

rsc.li/rsc-advances

\section{A highly sensitive electrochemical impedance immunosensor for indole-3-acetic acid and its determination in sunflowers under salt stress}

\author{
Haiyang Li, ${ }^{\text {ac }}$ Ye Hu, ${ }^{\text {ab }}$ Aixue Li, (D) *ab Xiaodong Wang, ${ }^{\text {ab }}$ Peichen Hou, ${ }^{\text {ab }}$ \\ Cheng Wang, ${ }^{\text {ab }}$ Ke Chen ${ }^{c}$ and Chunjiang Zhao*ab
}

\begin{abstract}
As an important phytohormone, indole-3-acetic acid (IAA) plays an essential role during the whole process of growth and development of plants. In this work, a novel label-free electrochemical impedance immunosensor for IAA determination has been developed. A three-dimensional PAMAM dendrimer was applied to improve greatly the immobilization capacity of antibodies for IAA on a gold electrode. Gold nanoparticles (AuNPs) were used to further improve the sensitivity of the biosensor by forming complexes with anti-IAA antibodies. The developed impedance immunosensor shows ultra-sensitivity, high selectivity and good reliability for IAA detection. The IAA extracted from the stems of sunflowers under different salt stress conditions was successfully detected using the immunosensor. The results are in good agreement with those obtained by UPLC-MS and consistent with published data, thus confirming the practical value of the biosensor. To the best of our knowledge, this is the first electrochemical impedance immunosensor for IAA detection, which provides a promising detection platform for IAA. By replacing antibodies, this strategy can be easily used to detect other phytohormones.
\end{abstract}

\section{Introduction}

Phytohormones are small organic molecules synthesized in trace amounts by plants. As signal molecules, they have significant effects on the growth and development of plants at very low concentrations. Therefore, phytohormones have been a hot topic in plant physiology. As a ubiquitous phytohormone, indole-3-acetic acid (IAA) is important for the regulation of multiple physiological processes of plants, including plant growth, cell division, flowering, withering, and gene expression. ${ }^{1-3}$ It also plays an important role in the response and adaptation of plants under stressed conditions. ${ }^{4,5}$ In view of the important role of IAA, it is very necessary to perform precise quantitative analysis of IAA in plants.

Currently, there are a variety of techniques applied for IAA detection, such as liquid chromatography (LC), ${ }^{6}$ gas chromatography-mass $\quad$ spectrometry(GC-MS $){ }^{7} \quad$ chemiluminescence, ${ }^{8}$ capillary electrophoresis(CE), ${ }^{9}$ spectrofluorometric methods ${ }^{\mathbf{1 0}}$ radioimmunoassays, ${ }^{\mathbf{1 1}}$ etc. Although these techniques show good sensitivity and selectivity for IAA

\footnotetext{
${ }^{a}$ Beijing Research Center of Intelligent Equipment for Agriculture, Beijing Academy of Agriculture and Forestry Sciences, Beijing 100097, China

${ }^{b}$ National Engineering Research Center for Information Technology in Agriculture, Beijing Academy of Agriculture and Forestry Sciences, Beijing 100097, China. E-mail: liax@nercita.org.cn; zhaocj@nercita.org.cn

${ }^{c}$ School of Life Science and Engineering, Southwest University of Science and Technology, Mianyang 621000, China
}

detection, some of them require expensive instruments, or involve a tedious procedure, or involve some radioactive chemicals. Therefore, there is still considerable interest for developing simple, low-cost, sensitive and reliable methods for IAA detection.

Electrochemical biosensors have long been considered as one of the most appealing techniques as they have many advantages, such as high sensitivity, rapid response, portability, and low cost, etc. ${ }^{12-14}$ A variety of electrochemical protocols have been used to construct IAA biosensors. ${ }^{15-19}$ For instance, Gan et $a .^{20}$ developed an disposable electrochemical sensor by differential pulse voltammograms method, basing on the application of nanocomposites of reduced graphene oxide (rGO) and poly(safranine T) (PST). They obtained a detection limit of $5 \times 10^{-8} \mathrm{M}$. Based on the multi-wall carbon nanotubes film coated GC electrode, $\mathrm{Wu}$ et $a .^{21}$ reported an amperometric sensor for IAA detection. The detection limit was $2 \times 10^{-8} \mathrm{~mol} \mathrm{~L}^{-1}$. Electrochemical impedance spectroscopy (EIS) is a very useful technique for studying the modification process of electrodes. ${ }^{22}$ The EIS technique is not only simple and sensitive, but also requires no labeling of the analyte with some redox moieties. However, the EIS technique has never been used for developing IAA biosensors to date.

For developing sensitive electrochemical biosensors, the construction of molecularly organized and stable detecting layers is very important. The fourth generation polyamidoamine ( $\mathrm{G}_{4}$ PAMAM) dendrimer belongs to a highly branched and symmetrical polymer. It has unique chemical and structural 
properties, ${ }^{23}$ such as precise molecular structure, a large number of surface functional groups, and good biocompatibility, etc., so it is an ideal component for biosensor fabrication. ${ }^{24}$ It has been applied in the construction of biosensors for a variety of biomolecules. ${ }^{25,26}$ However, as far as we know, PAMAM has not been used in the construction of an electrochemical impedance biosensor for IAA detection.

In this work, a multilayered film including PAMAM was assembled on gold electrode. This highly branched PAMAM films increase the amount of immobilized antibody of IAA greatly. Gold nanoparticles (AuNPs) were also used to form anti-IAA-AuNPs complex to improve the sensitivity of the biosensor. Based on the application of PAMAM and the anti-IAA-AuNPs complex, this electrochemical impedance immunosensor exhibits ultrasensitivity, high selectivity and good reliability for IAA detection. Furthermore, IAA in the stem of sunflowers under different salt stress was detected using the prepared immunosensor, confirming the practical applicability of the sensor.

\section{Experimental}

\subsection{Materials}

G4 ethylenediamine (EDA) core poly(amidoamine) (PAMAM) dendrimer was purchased from Aldrich. Monoclonal antibody against IAA, cysteamine (AET), indole-3-acetic acid (IAA), citric acid, abscisic acid (ABA), succinic acid, salicylic acid (SA), malic acid, jasmonic acid (JA) and glutaraldehyde (GA) were purchased from Sigma-Aldrich (Germany). Bovine serum albumin (BSA) was obtained from Beijing BioDee BioTech Corporation Ltd (China). Gold nanoparticles was purchased from Nanjing XFNano Material Tech Co., Ltd. (Nanjing, China). The average diameters were about $15 \mathrm{~nm}$. All other reagents were of analytical reagent grade. Ultrapure water was used for the whole experiment.

\subsection{Preparation of anti-IAA-AuNPs complex}

The anti-IAA-AuNPs complex was produced by the same procedure described elsewhere. ${ }^{\mathbf{1 6 , 2 7}}$ The $\mathrm{pH}$ of colloidal AuNPs solutions was maintained at 7.4 by adding $10 \mathrm{mM} \mathrm{K}_{2} \mathrm{CO}_{3}$ before adding the antibody. $100 \mu \mathrm{L}$ anti-IAA antibodies $\left(1 \mathrm{mg} \mathrm{mL}^{-1}\right)$ were mixed with $400 \mu \mathrm{L}$ colloids AuNPs solutions, and followed by shaking for $5 \mathrm{~min}$. Then, BSA was added (concentration of $1 \%$ ) and the mixture was incubated overnight at $4{ }^{\circ} \mathrm{C}$. The unbound antibody was eliminated from the solution by centrifugation at $12000 \mathrm{rpm}$ for $20 \mathrm{~min}$. Then the resulted anti-IAAAuNPs were re-dispersed in $1 \mathrm{~mL}$ PBS $(0.1 \mathrm{M}, \mathrm{pH}=7.4)$ and stored at $4{ }^{\circ} \mathrm{C}$ until use.

\subsection{Fabrication of the electrochemical immunosensor}

The gold disk electrode ( $d=2 \mathrm{~mm}, \mathrm{CH}$ instruments, China) was polished with alumina powder $(0.3$ and $0.05 \mu \mathrm{m})$. After that, it was sonicated in double-distilled water and ethanol for $3 \mathrm{~min}$ successively. The electrode was electrochemically cleaned in $1 \mathrm{M}$ $\mathrm{H}_{2} \mathrm{SO}_{4}$. The cleaned gold electrode was put in $10 \mathrm{mM}$ AET and incubated for 8 hours at $4{ }^{\circ} \mathrm{C}$, assembling the AET molecules on the electrode through $\mathrm{Au}-\mathrm{S}$ bonds. ${ }^{28}$ After washing by ethanol and water, the AET modified electrode was incubated in 5\% GA for 4 hours at $4{ }^{\circ} \mathrm{C}$. Subsequently, the modified electrode was put in PAMAM ( $10 \%$ in methanol) for 16 hours at $4{ }^{\circ} \mathrm{C}$. The immobilization of PAMAM is due to that the amino group of AET and dendrimer can be covalently bonded to the aldehyde group of GA. The electrode was then immersed in 5\% GA for $4 \mathrm{~h}$. After that, the electrode was immersed in antibody-AuNPs for 8 hours at $4{ }^{\circ} \mathrm{C}$. In order to eliminate the effect of nonspecific binding and residual reactive groups, the antibody-modified electrode was treated with $1 \%$ BSA for $30 \mathrm{~min}$. The fabrication process of the electrochemical immunosensor is displayed in Scheme 1.

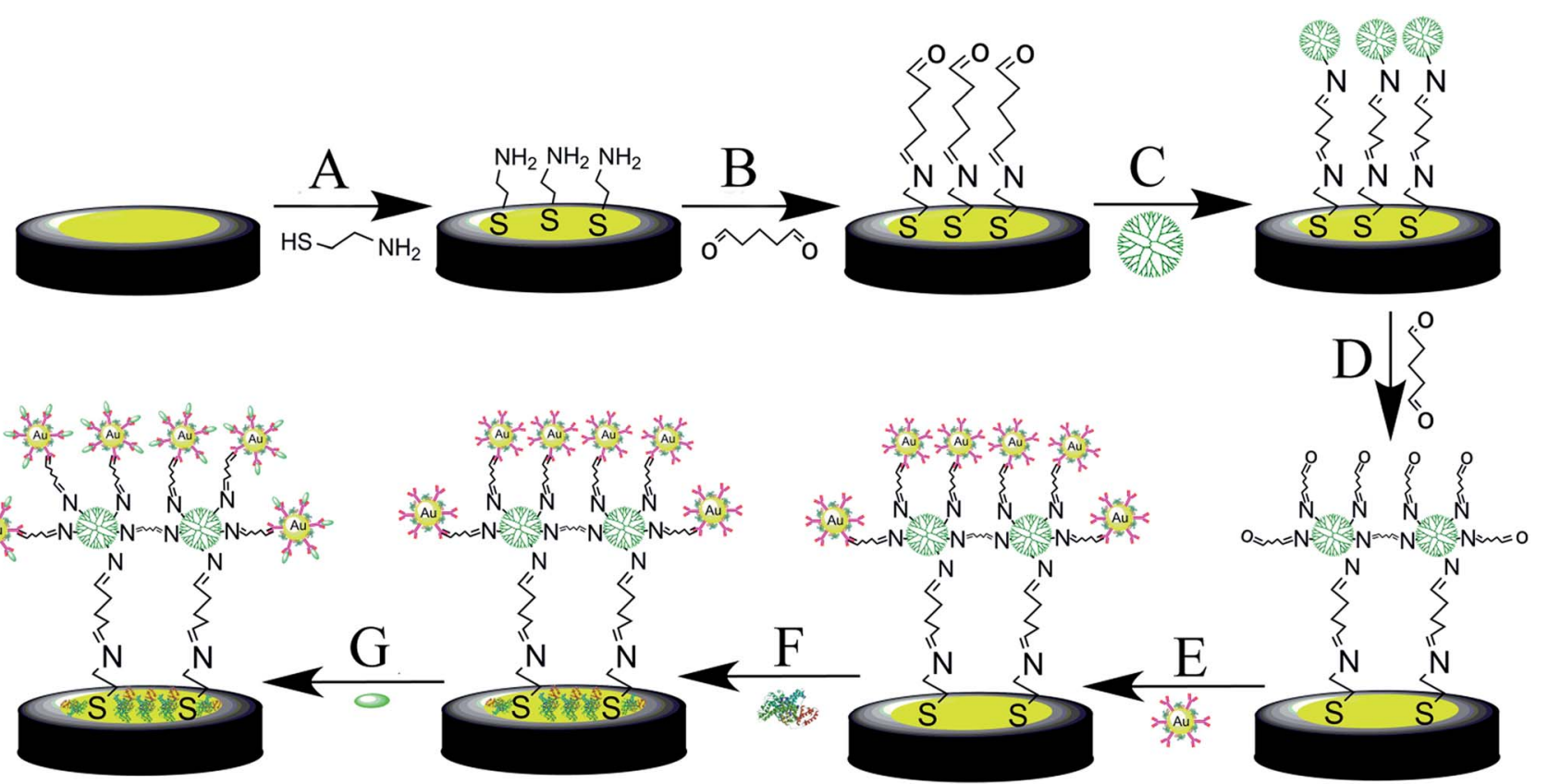

Scheme 1 The schematic illustration of the fabrication process of the IAA immunosensor. (A) $10 \mathrm{mM} \mathrm{AET} \mathrm{in} \mathrm{ethanol,} 8 \mathrm{~h}$; (B) $5 \% \mathrm{GA}, 4 \mathrm{~h}$; (C) $10 \%$ methanolic solution of PAMAM, 16 h; (D) 5\% GA, 4 h; (E) anti-IAA-AuNPs complex, 8 h; (F) 1\% BSA in PBS, 30 min; (G) IAA in PBS, 1 h. 


\subsection{Electrochemical measurements}

PGSTAT302N AUTOLAB electrochemical analyzer system (Eco Chemie B. B. Netherlands) was applied for the electrochemical experiments. All the electrochemical measurements were performed in 0.1 M PBS ( $\mathrm{pH} 7.4$ ) containing $5 \mathrm{mM} \mathrm{Fe}(\mathrm{CN})_{6}{ }^{3-/ 4-}$ as redox probe at room temperature. A three-electrode system was applied. $\mathrm{Ag} / \mathrm{AgCl}$ electrode (saturated $\mathrm{KCl}$ ) was applied as reference electrode. Pt plate (area, $2 \times 1 \mathrm{~cm}^{2}$ ) was used as counter electrode. A modified gold electrode was used as working electrode. The impedance spectra were measured from $100 \mathrm{kHz}$ to $100 \mathrm{mHz}$, and the voltage amplitude is $5 \mathrm{mV}$.

\subsection{X-ray photoelectron spectroscopy (XPS) measurements}

XPS measurement was performed on ESCALAB 250Xi spectrometer (Thermo Fisher, USA), and Al $\mathrm{K} \alpha$ excitation was applied. The peak positions of the elements were calibrated by $\mathrm{C}$ 1s $(284.5 \mathrm{eV})$. Cleaned gold substrates which were modified in the similar manner to the gold electrode were used for the XPS measurement.

\subsection{Preparation of plant material samples}

The seeds of sunflower (LD5009) were obtained from Beijing Kafry Technology Co., LTD. Evenly sized, healthy sunflower seeds were selected, sterilized, and soaked. Then they were cultured in an illuminating incubator $(10 \mathrm{~h} / 14 \mathrm{~h}$ of light/dark period, $25 \pm 2{ }^{\circ} \mathrm{C}$ and $60 \%$ humidity). After germination, the seedlings with consistent growth were selected and transferred into Hoagland solution. When the seedlings grew to 15 days, three levels of salts were added in Hoagland's solution, i.e. 0 (control), 50, and $100 \mathrm{mM}$. After 10 hours of salt treatment, the stem samples of the seedlings were frozen in liquid nitrogen immediately.

IAA was extracted and purified from the stem of sunflower seedlings according to previous reports with some modification. ${ }^{16,20}$ The samples were ground with liquid nitrogen. $1 \mathrm{~g}$ of ground powder was added $2 \mathrm{~mL}$ precooled methanol $\left(4^{\circ} \mathrm{C}, 80 \%\right)$. After stirring, the powder was extracted overnight at $4{ }^{\circ} \mathrm{C}$. The solution was added $1 \mathrm{~mL}$ of $\mathrm{CHCl}_{3}$. Then it was vortexed for $10 \mathrm{~s}$, following shaked at $900 \mathrm{rpm}$ for $4 \mathrm{~min}$ at $4{ }^{\circ} \mathrm{C}$. After centrifuging (12 $000 \mathrm{rpm}, 5 \mathrm{~min}, 4^{\circ} \mathrm{C}$ ), the supernatant was collected and blow-dried with nitrogen at room temperature. Finally, for the electrochemical immunosensor, the sample was dissolved in $1 \mathrm{~mL}$ of $10 \mathrm{mM}$ PBS (pH 7.4). For UPLC-MS measurements (Water Acquity I-Class, Waters Corporation, MA, US; Thermo Q-Exactive, Thermo Scientific, MA, US), the sample was dissolved in $1 \mathrm{~mL}$ of methanol and passed through a $0.22 \mu \mathrm{m}$ microporous membrane.

\section{Results and discussion}

\subsection{Characterizations of the fabrication of the} immunosensor

In this study, EIS was carried out to study the electron transfer resistance due to the change of each modification step on the electrode. ${ }^{29,30}$ Fig. 1A illustrates the Nyquist plots of a bare $\mathrm{Au}$ electrode (a), GA/AET/Au (b), GA/PAMAM/GA/AET/Au (c), and
AuNPs-anti-IAA/GA/PAMAM/GA/AET/Au (d) in 5 mM Fe(CN) ${ }_{6}{ }^{3-/ 4-}$ solution. The impedance plots obtained present a Faradaic system. It includes a diffusion-controlled section at lower frequency related to the Warburg impedance, and a semicircle region at higher frequency related to the electron transfer resistance. ${ }^{31}$ For fitting the results of impedance spectroscopy, an equivalent circuit (the inset in Fig. 1A) was applied. In this circuit, $R_{\mathrm{S}}$ represents the resistance of the solution, $W$ represents the Warburg impedance, $R_{\mathrm{ct}}$ represents the resistance for charge transfer, and CPE (constant phase element) represents the capacitance of the double layer. CPE was used instead of a capacitor due to the non-homogeneous nature of the electrode surface, and it led to a better fitting of the experimental data. The CPE and Warburg impedance can be expressed by

$$
\begin{aligned}
Z_{\mathrm{CPE}} & =\frac{1}{Y_{0}(j \omega)^{n}} \\
Z_{\mathrm{W}} & =\frac{1}{Y_{0} \sqrt{j \omega}}
\end{aligned}
$$

where $j$ is the imaginary number, $\omega$ is the angular frequency, and $0<n<1$. When $n$ is closer to 1 , CPE becomes more capacitive.

The parameters obtained by fitting are summarized in Table 1. After the AET layer was formed on the electrode, the $R_{\mathrm{ct}}$ value is decreased. Because the AET molecule is positively charged under the experimental conditions ( $\mathrm{pH}$ 7.4) and it will attract the negative redox probes (data not shown). But after the modification of GA layer on the electrode (curve b), the $R_{\mathrm{ct}}$ is increased to $101.2 \Omega$ due to the formation of AET layer and GA layer and the neutralization of the GA molecules. When the PAMAM and the second GA layer were assembled on the electrode, the $R_{\mathrm{ct}}$ value is increased to $695.6 \Omega$ (curve c) because of the compact structure of the assembled films. The $R_{\mathrm{ct}}$ value (curve d) increased further after the modification of the AuNPsanti-IAA on the electrode, which is due to the steric hindrance of the AuNPs-anti-IAA complex. ${ }^{32,33}$

The assembly process of the electrode was also characterized by $\mathrm{CV}$ technique. As shown in Fig. 1C, compare with the bare gold electrode (curve a), after the AET and GA layer (curve b) was formed on the electrode, the peak current was decreased and the separation of redox potential was increased. When the PAMAM layer, the second GA layer, and the anti-IAA-AuNP complex were further assembled on the electrode in turn, the peak current was decreased further. And the reversibility of redox peaks becomes worse, because the electron transfer between the redox probe and the electrode surface was hindered by the layer-by-layer modification of the electrode. This result also confirms the successful fabrication of the immunosensor.

X-ray photoelectron spectroscopy (XPS) was used to characterize the elements of the multicomponent membrane. ${ }^{34}$ Fig. $1 \mathrm{D}$ illustrates the XPS spectra of the $\mathrm{N}$ 1s region. The peak of $\mathrm{N} 1 \mathrm{~s}$ appears at $399.5 \mathrm{eV}$. With the layer by layer modification on the electrode, the $\mathrm{N}$ 1s intensities increased monotonously. The result is as expected, as nitrogen content is contained in AET, PAMAM and antibody molecules. Therefore, the XPS result 

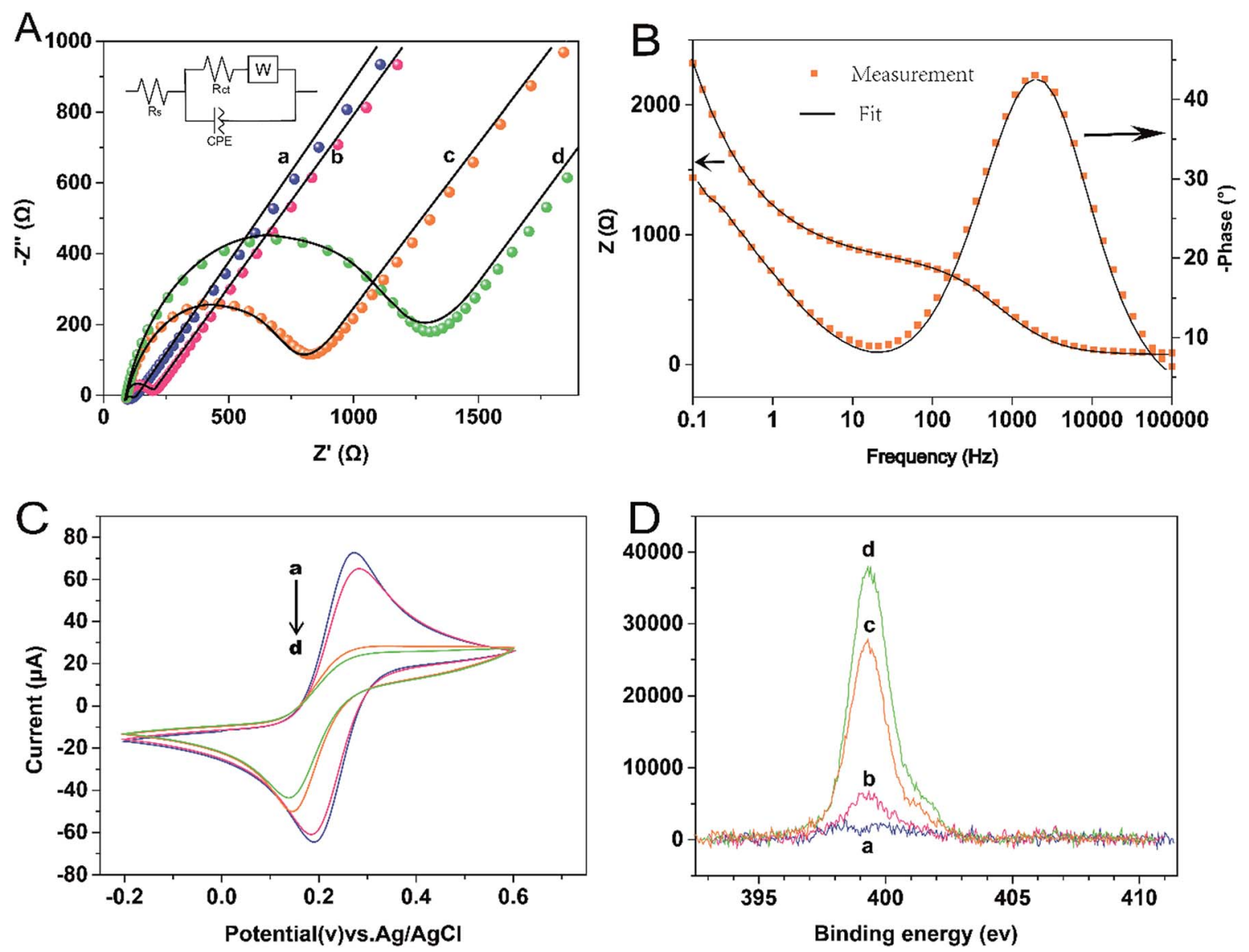

Fig. 1 (A) Measured (point) and simulated (line) complex impedance plot of the modified gold electrode in $0.01 \mathrm{M} \mathrm{PBS}$ (pH 7.4) containing $5 \mathrm{mM}$ $\left[\mathrm{F}_{\mathrm{e}}(\mathrm{CN})_{6}\right]^{3-/ 4-}$ : (a) bare Au, (b) GA/AET/Au, (c) GA/PAMAM/GA/AET/Au and (d) AuNPs-anti/GA/PAMAM/GA/AET/Au. Inset was the equivalent circuit model used to fit the impedance data. $R_{\mathrm{s}}$, solution resistance; $R_{\mathrm{ct}}$, resistance of charge transfer; $C P E$, constant phase element; $W$, Warburg impedance. (B) Bode diagrams of the impedance spectra of measured and fitted curve for GA/PAMAM/GA/AET/Au (curve $c$ in A) in the process of modification. (C) Cyclic voltammograms of the modified gold electrode in $0.01 \mathrm{M}$ PBS $(\mathrm{pH} 7.4)$ containing $5 \mathrm{mM}\left[\mathrm{F}_{\mathrm{e}}(\mathrm{CN})_{6}\right]^{3-/ 4-}$. (D) High resolution XPS spectra of $\mathrm{N}$ 1s of the gold substrate after various surface modification steps. For (C) and (D), a, b, c, d were the same as in (A).

further demonstrates that each organic layer was successfully assembled on the surface of the gold substrate.

\subsection{Analytical performance of the immunosensor}

To evaluate the response of the immunosensor to IAA, the immunosensor was incubated with different concentrations of IAA for 1 hour. Then, the EIS measurements was carried out in $5 \mathrm{mM} \mathrm{Fe}(\mathrm{CN})_{6}{ }^{3-/ 4-}$. As shown in Fig. 2, with the increase of the

Table 1 Parameter values of the equivalent circuit elements for the modification process of the gold electrode. $a, b, c, d$ were the same as in Fig. $1 \mathrm{~A}$

\begin{tabular}{lllrrl}
\hline & & \multicolumn{2}{l}{ CPE } & & \\
\cline { 3 - 4 } & & $Y_{0}$ & & & \\
& $R_{\mathrm{S}}(\Omega)$ & $\left(\mu \mathrm{Mhos} \mathrm{s}^{n}\right)$ & $n$ & $R_{\mathrm{ct}}(\Omega)$ & $\begin{array}{l}\text { Warburg impedance } \\
\left(\mu \mathrm{Mhos}^{-1} \mathrm{~s}^{-0.5}\right)\end{array}$ \\
\hline $\mathrm{a}$ & 72.1 & 1.74 & 0.801 & 30.3 & 789 \\
$\mathrm{~b}$ & 74.4 & 1.06 & 0.878 & 101.2 & 759 \\
$\mathrm{c}$ & 73.9 & 1.81 & 0.832 & 695.6 & 738 \\
$\mathrm{~d}$ & 74.1 & 1.51 & 0.844 & 1075.9 & 736
\end{tabular}

IAA concentrations, the $R_{\mathrm{ct}}$ values increase. The change of the $R_{\text {ct }}$ value $\left(\Delta R_{\mathrm{ct}}\right)$ is proportional to the logarithm value of IAA concentration in the range of $10 \mathrm{pg} \mathrm{mL} L^{-1}-10 \mu \mathrm{g} \mathrm{mL}^{-1}$ (Fig. 3a). The linear regression equation is $\Delta R_{\mathrm{ct}}=105.9 \times \log C+1294.9$ with the correlation coefficient of 0.998 . The detection limit was $4.62 \mathrm{pg} \mathrm{mL}^{-1}(\mathrm{~S} / \mathrm{N}=3)$. The sensitivity of the developed impedance immunosensor is superior to some other methods for IAA detection based on other techniques, such as HPLC-MS/ MS, ${ }^{35}$ chemiluminescence, ${ }^{36}$ capillary electrophoresis,${ }^{37}$ etc. The sensitivity of the sensor is also higher than most electrochemical sensors existed for IAA. ${ }^{15-20,38,39}$

Two other types of immunosensors, one without the modification of PAMAM (sensor B) and one without the application of $\mathrm{Au}$ NP (sensor C), were also prepared to compare with the developed immunosensor (sensor A). Sensor B and C were also incubated with different concentrations of IAA. As shown in Fig. 3, the calibration curve for sensor B is $\Delta R_{\mathrm{ct}}=52.2 \times \log C+$ 543 and the linear range is $100 \mathrm{pg} \mathrm{mL}^{-1}-1 \mu \mathrm{g} \mathrm{mL}^{-1}$. The detec-

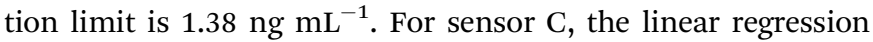
equation is $\Delta R_{\mathrm{ct}}=38.5 \times \log C+423.1$ and its linear range is

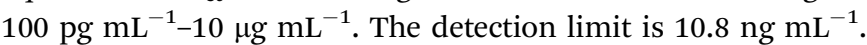




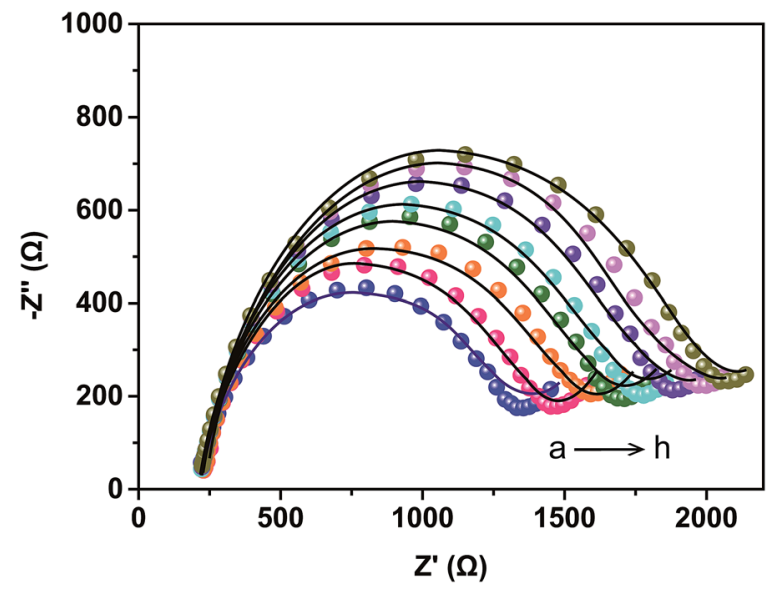

Fig. 2 Measured (point) and simulated (line) complex impedance plot of the prepared immunosensor after incubation with IAA (a) 0 , (b) $10 \mathrm{pg} \mathrm{mL}^{-1}$, (c) $100 \mathrm{pg} \mathrm{mL}^{-1}$, (d) $1 \mathrm{ng} \mathrm{mL}^{-1}$, (e) $10 \mathrm{ng} \mathrm{mL}^{-1}$, (f) $100 \mathrm{ng} \mathrm{mL}^{-1}$, (g) $1 \mu \mathrm{g} \mathrm{mL}{ }^{-1}$, (h) $10 \mu \mathrm{g} \mathrm{mL}^{-1}$ in the presence of $5 \mathrm{mM}\left[\mathrm{F}_{\mathrm{e}}(\mathrm{CN})_{6}\right]^{3-/ 4-}$.

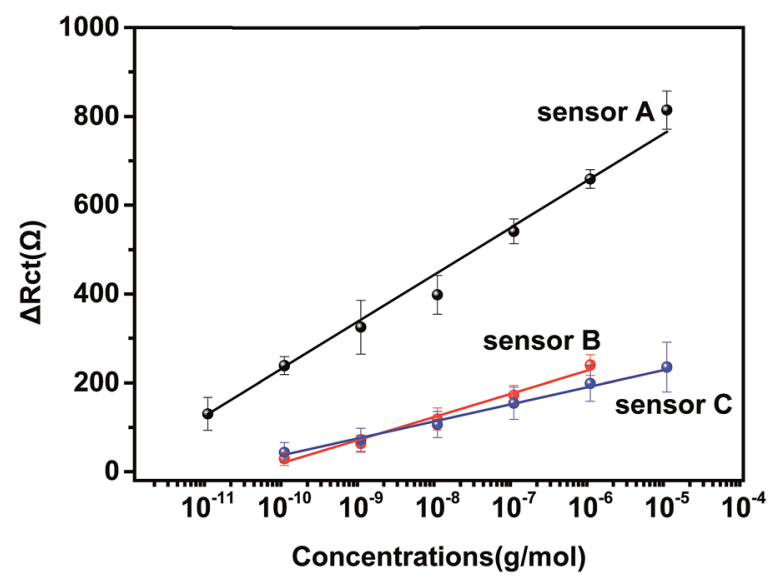

Fig. 3 Calibration curve of three types of immunosensors for IAA. The concentrations used were the same as those in Fig. 2. Sensor A: AuNPs-anti/GA/PAMAM/GA/AET/Au, sensor B: anti-IAA/GA/PAMAM/ GA/AET/Au, sensor C: AuNPs-anti-IAA/GA/AET/Au.

The performance of sensor B and C were significantly lower than sensor A. This result indicated that the unique properties of PAMAM and the application of anti-IAA-AuNP complex improved greatly the performance of the impedance immunosensor.

Selectivity is also important for sensor application. To evaluate the selectivity of the immunosensor, succinic acid $\left(1 \mathrm{ng} \mathrm{mL} \mathrm{m}^{-1}\right)$,

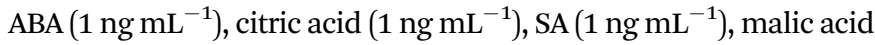
$\left(1 \mathrm{ng} \mathrm{mL}^{-1}\right)$, JA $\left(1 \mathrm{ng} \mathrm{mL}{ }^{-1}\right)$ and $10 \mathrm{pg} \mathrm{mL}^{-1}$ IAA was used to react with the immunosensor respectively. As shown in Fig. 4, although the concentration of the interference species are 100 times as that of the IAA concentration, the obtained $\Delta R_{\mathrm{ct}}$ value of the interference species is too low to affect the detection of IAA. This result demonstrates that the immunosensor has high selectivity.

As for the reproducibility of sensor, intra-assay and inter-assay experiments were carried out. The intra-assay was estimated by assaying $1 \mathrm{ng} \mathrm{mL} \mathrm{mL}^{-1}$ IAA concentration five times, resulting in a variation coefficient of $4.1 \%$. The variation coefficient for the

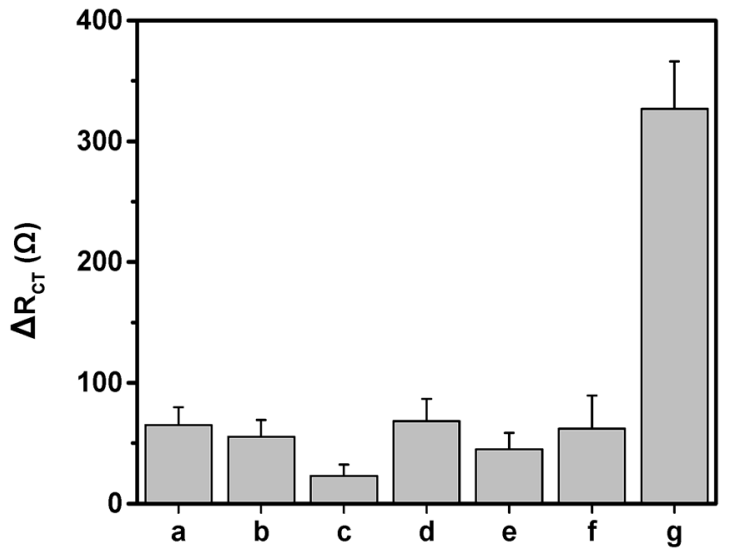

Fig. 4 EIS response of the immunosensor to some interference species: a (ABA), b (succinic acid), c (citric acid), d (SA), e (malic acid), f (JA) and $g(I A A)$. The concentration of ABA, succinic acid, SA, malic acid, JA and citric acid was $1 \mathrm{ng} \mathrm{mL}^{-1}$, the concentration of IAA was $10 \mathrm{pg} \mathrm{mL}^{-1}, n=6$.

inter-assay was $4.9 \%$ using five immunosensors fabricated by the same process. These results indicate good reproducibility of the immunosensor. There is still $89.6 \%$ sensing ability remained for the immunosensor after storage at $4{ }^{\circ} \mathrm{C}$ for two weeks, indicating that the immunosensor is highly stable.

\subsection{Detection of IAA in sunflowers under salt stress}

Salt stress is one of the main limit factors for the growth and development of plant. ${ }^{40}$ Phytohormones play crucial roles in the process of plant resistance to stress response. ${ }^{41,42}$ In our work, in order to testify the practical application of the immunosensor, IAA in the stem samples of sunflowers under different levels of salt stress was determined by the developed immunosensor. The concentration of IAA in the stem part of sunflowers is $15.31 \pm 0.51 \mathrm{ng} \mathrm{mL}^{-1}, 6.65 \pm 0.32 \mathrm{ng} \mathrm{mL}^{-1}$, and $4.14 \pm 0.15 \mathrm{ng} \mathrm{mL}{ }^{-1}$ for $0 \mathrm{mM}, 50 \mathrm{mM}$ and $100 \mathrm{mM}$ of $\mathrm{NaCl}$ treatment, respectively. Our result showed that with the increasing concentrations of salt, the IAA level was decreased in the stem of sunflowers. This result is consistent with previous studies, which showed that the inhibition of salinity on plant growth may have a relation with the decreasing levels of IAA. ${ }^{15,42,43}$ In addition, as shown in Table 2, the detection results of the immunosensor were in good aggrement with those obtained by UPLC-MS. These results confirm that the

Table 2 Comparation of the results obtained by UPLC-MS and the developed immunosensor for detecting IAA in the stem samples of sunflowers under salt stress

\begin{tabular}{lrll}
$\begin{array}{l}\mathrm{NaCl} \\
\text { concentrations } \\
(\mathrm{mM})\end{array}$ & $\begin{array}{c}\text { UPLC-MS } \\
\left(\mathrm{ng} \mathrm{mL} \mathrm{mL}^{-1}\right)\end{array}$ & $\begin{array}{l}\text { Immunosensor } \\
\left(\mathrm{ng} \mathrm{mL}^{-1}\right)\end{array}$ & $\begin{array}{l}\text { Relative } \\
\text { deviation }(\%)\end{array}$ \\
\hline 0 & $12.20 \pm 1.91$ & $15.31 \pm 0.51$ & $4.66 \%$ \\
50 & $5.16 \pm 0.70$ & $6.65 \pm 0.32$ & $7.42 \%$ \\
100 & $3.34 \pm 0.90$ & $4.14 \pm 0.15$ & $2.80 \%$
\end{tabular}


developed immunosensor can reliably detect IAA in real samples.

\section{Conclusions}

In summary, for the first time, we developed a label-free impedance immunosensor for IAA based on the application of PAMAM and the anti-IAA-AuNPs complex. This electrochemical impedance immunosensor exhibited ultra-sensitivity, high selectivity and good reliability for IAA detection. The applicability of the fabricated immunosensor was also confirmed by detecting IAA in the stem of sunflowers under different salt stress. The proposed approach provides a promising detection platform for IAA detection, and this strategy could easily extend to detect other phytohormones by replacing the antibody.

\section{Conflicts of interest}

There are no conflicts to declare.

\section{Acknowledgements}

The authors are thankful for the fundings from Scientific and Technological Innovation Team of Beijing Academy of Agricultural and Forestry Sciences (JNKYT201604), and the National Natural Science Foundation of China (Grant No.61571443 and No.51402063).

\section{References}

1 H. T. Liu, Y. F. Li, T. G. Luan, C. Y. Lan and W. S. Shu, Chromatographia, 2007, 66, 515-520.

2 Z. Xi, Z. Zhang, Y. Sun, Z. Shi and W. Tian, Talanta, 2009, 79, 216-221.

3 Z. Ma, L. Ge and A. S. Y. Lee, Anal. Chim. Acta, 2008, 610, 274281.

4 S. Fahad, S. Hussain, A. Matloob, F. A. Khan, A. Khaliq, S. Saud, S. Hassan, D. Shan, F. Khan, N. Ullah, M. Faiq, M. R. Khan, A. K. Tareen, A. Khan, A. Ullah, N. Ullah and J. Huang, Plant Growth Regul., 2014, 75, 391-404.

5 F. Eyidogan, M. Oz, M. Yucel and H. Oktem, in Phytohormones and Abiotic Stress Tolerance in Plants, Springer, 2012, pp. 1-48.

6 H. T. Liu, Y. F. Li, T. G. Luan, C. Y. Lan and W. S. Shu, Chromatographia, 2007, 66, 515-520.

7 S. Pollmann, A. Müller, M. Piotrowski and E. W. Weiler, Planta, 2002, 216, 155-161.

8 H. Xu, Z. Mei, Z. Wang and Y. He, Spectrochim. Acta, Part A, 2012, 95, 114-119.

9 H. Chen, X. F. Guo, H. S. Zhang and H. Wang, J. Chromatogr. B: Anal. Technol. Biomed. Life Sci., 2011, 879, 1802-1808.

10 Y. N. Li, H. L. Wu, S. H. Zhu, J. F. Nie, Y. J. Yu, X. M. Wang and R. Q. Yu, Anal. Sci., 2009, 25, 83-88.

11 A. Madej and P. Häggblom, Physiol. Plant., 1985, 64, 389-392.

12 Y. Guo, Q. Wang, Z. Wang, X. Chen, L. Xu, J. Hu and R. Pei, Sens. Actuators, B, 2015, 214, 50-55.

13 J. Fan, K. Qi, L. Zhang, H. Zhang, S. Yu and X. Cui, ACS Appl. Mater. Interfaces, 2017, 9, 18008-18014.
14 J. Sun, B. Wang, X. Zhao, Z.-J. Li and X. Yang, Anal. Chem., 2016, 88, 1355-1361.

15 L. J. Sun, X. J. Liu, L. Gao, Y. N. Lu, Y. B. Li, Z. Q. Pan, N. Bao and H. Y. Gu, Anal. Lett., 2015, 48, 1578-1592.

16 Y. Zhou, Z. Xu, M. Wang, X. Meng and H. Yin, Electrochim. Acta, 2013, 96, 66-73.

17 F. Liu, J. Tang, J. Xu, Y. Shu, Q. Xu, H. Wang and X. Hu, Biosens. Bioelectron., 2016, 86, 871-878.

18 L. Lu, Y. Yu, H. Zhou, P. Li, Y. Peng, W. Wang and H. He, Int. J. Electrochem. Sci., 2016, 11, 2392-2400.

19 S. Lu, L. Bai, Y. Wen, M. Li, D. Yan, R. Zhang and K. Chen, J. Solid State Electrochem., 2014, 19, 2023-2037.

20 T. Gan, C. Hu, Z. Chen and S. Hu, Talanta, 2011, 85, 310-316.

21 K. Wu, Y. Sun and S. Hu, Sens. Actuators, B, 2003, 96, 658662.

22 D. Feng, L. Li, X. Fang, X. Han and Y. Zhang, Electrochim. Acta, 2014, 127, 334-341.

23 C. S. Rao Vusa, V. Manju, S. Berchmans and P. Arumugam, RSC Adv., 2016, 6, 33409-33418.

24 E. B. Bahadır and M. K. Sezgintürk, Talanta, 2016, 148, 427-438.

25 R. Akter, B. Jeong, Y. M. Lee, J. S. Choi and M. A. Rahman, Biosens. Bioelectron., 2017, 91, 637-643.

26 B. Borisova, A. Sánchez, S. Jiménez-Falcao, M. Martín, P. Salazar, C. Parrado, J. M. Pingarrón and R. Villalonga, Sens. Actuators, B, 2016, 232, 84-90.

27 H. Yin, Z. Xu, Y. Zhou, M. Wang and S. Ai, Analyst, 2013, 138, 1851-1857.

28 Y. Liu, Y. Li, S. Liu, J. Li and S. Yao, Biomaterials, 2004, 25, 5725-5733.

29 A. X. Li, Y. Ma, F. Yang and X. R. Yang, Appl. Surf. Sci., 2007, 253, 6103-6108.

30 P. K. Brahman, L. Suresh, K. R. Reddy and J. S. Bondili, RSC Adv., 2017, 37898-37907.

31 J. S. Daniels and N. Pourmand, Electroanalysis, 2007, 12, 1239-1257.

32 J. Lin, R. Wang, P. Jiao, Y. Li, Y. Li, M. Liao, Y. Yu and M. Wang, Biosens. Bioelectron., 2015, 67, 546-552.

33 B. Kavosi, A. Salimi, R. Hallaj and K. Amani, Biosens. Bioelectron., 2014, 52, 20-28.

34 F. Tielens, D. Costa, V. Humblot and C. M. Pradier, J. Phys. Chem. C, 2008, 112, 182-190.

35 S. Giannarelli, B. Muscatello, P. Bogani, M. M. Spiriti, M. Buiatti and R. Fuoco, Anal. Biochem., 2010, 398, 60-68.

36 X. Hun, Z. Mei, Z. Wang and Y. He, Spectrochim. Acta, Part A, 2012, 95, 114-119.

37 H. Chen, X. F. Guo, H. S. Zhang and H. Wang, J. Chromatogr. B: Anal. Technol. Biomed. Life Sci., 2011, 879, 1802-1808.

38 T. Gan, C. Hu, Z. Chen and S. Hu, Talanta, 2011, 85, 310-316. 39 B. Sun, L. Chen, Y. Xu, M. Liu, H. Yin and S. Ai, Biosens. Bioelectron., 2014, 51, 164-169.

40 K. Aghaei and S. Komatsu, Front. Plant Sci., 2013, 4, 8.

41 Z. Peleg and E. Blumwald, Curr. Opin. Plant Biol., 2011, 14, 290-295.

42 M. G. Javid, A. Sorooshzadeh, F. Moradi, et al., Aust. J. Crop Sci., 2011, 32, 726-734.

43 Y. Wang, S. Mopper and K. H. Hasenstein, J. Chem. Ecol., 2001, 27, 327-342. 